\title{
OPEN ZBED6 regulates Igf2 expression partially through its regulation of miR483 expression
}

\author{
Rakan Naboulsi ${ }^{1}$, Mårten Larsson ${ }^{1}$, Leif Andersson ${ }^{1,2,3 凶}$ \& Shady Younis ${ }^{1,4,5 凶}$
}

The expression of $\lg f 2$ in mammals shows a complex regulation involving multiple promoters and epigenetic mechanisms. We previously identified a novel regulatory mechanism based on the interaction between the transcriptional factor ZBED6 and Igf2 intron. Disruption of the ZBED6-Igf2 interaction leads to a dramatic up-regulation of IGF2 expression postnatally. In the current study we characterize an additional layer of regulation involving miR 483 encoded by another $\lg f 2$ intron. We found a highly significant up-regulation of $m i R 483$ expression when the ZBED6-Igf2 axis is disrupted in transgenic mice. Furthermore, CRISPR/Cas9 mediated knock-out of miR483 in C2C12 myoblast cells, both wild-type and cells with disrupted ZBED6-Igf2 axis (Igf $2^{\mathrm{dGGCT}}$ ), resulted in down-regulation of Igf 2 expression and a reduced proliferation rate. This was further validated using $m i R 483$ mimics and inhibitors. RNA-seq analysis revealed a significant enrichment of genes involved in the PI3K-Akt signaling pathway among genes down-regulated in $m i R 483^{-1-}$ cells, including Igf 2 down-regulation. The opposite pattern was observed in $I g \mathrm{f}^{\mathrm{dGGCT}}$ cells, where $\lg f 2$ is up-regulated. Our data suggest a positive feedback between miR483 and Igf2 promoter activity, strongly affecting how ZBED6 controls Igf2 expression in various cell types.

Insulin-like growth factor 2 (Igf2) is paternally expressed in mammals and is a crucial fetal growth factor ${ }^{1}$. Transcriptional regulation of Igf2 is complex and occurs at different levels starting from its genomic locus, which includes the $H 19$ gene, an imprinted control region (ICR), and a set of enhancers. On the maternal allele, the unmethylated ICR restricts the access of enhancers to the Igf2 gene, which is therefore silenced, and directs them to promote $\mathrm{H} 19$ expression. In contrast, the heavily methylated ICR on the paternal allele promotes Igf2 transcription and leads to the corresponding silencing of $H 19$ expression ${ }^{1,2}$.

Genetic studies in domestic pigs revealed that Igf2 causes a major Quantitative Trait Locus (QTL) affecting muscle growth and fat deposition ${ }^{3}$, demonstrating that IGF2 also plays an important role for postnatal growth. The identification of a single base change in Igf2 intron 3 of pigs as the causal mutation of this QTL led to the discovery that this mutation disrupts the binding site for a previously unknown transcription factor, ZBED6 ${ }^{4}$. ZBED6 is a transcriptional repressor with the specific binding motif 5'-GCTCGC-3', present in an intron of Igf2 and at thousands of other putative binding sites in the mammalian genome $e^{4-8}$. Recently, we generated $Z$ bed 6 knock-out mice (Zbed6-KO) as well as Igf2 knock-in mice (Igf2-KI) carrying exactly the same single base change found in mutant pigs ${ }^{9}$. Both lines showed a $>$ tenfold upregulation of Igf2 expression at the mRNA level in skeletal muscle, heart and kidney, an eightfold upregulation of circulating IGF2 and increased muscle growth and enlarged heart, kidney and liver?

Igf2 shows upregulated expression in both pediatric tumors such as Wilm's tumor, hepatoblastoma and rhabdomyosarcoma, and adult tumors including colorectal cancer, hepatocellular carcinoma and breast cancer ${ }^{10}$. Loss of imprinting and biallelic expression of $I g f 2$ is a common finding in tumors. The overexpression of $I g f 2$ in these tumors are usually much higher than can be explained by loss-of imprinting only. However, the association to $m i R 483$, a gene hosted in an intron of $\operatorname{Igf2}$ and co-expressed with $I g f 2$, plays a prominent role for enhanced Igf2 expression in tumors ${ }^{11}$. miR483-3p and miR483-5p, the two miRNA products of miR483, are overexpressed in many cancers ${ }^{11,12}$. The transcription of miR483 is activated by the transcriptional activator USF $1^{10,13}$ that acts as a mediator between the oncoprotein $\beta$-catenin (CTNNB1) and the miR483 locus $^{14}$ in a glucose-dependent manner

\footnotetext{
${ }^{1}$ Science for Life Laboratory, Department of Medical Biochemistry and Microbiology, Uppsala University, 751 23 Uppsala, Sweden. '2Department of Animal Breeding and Genetics, Swedish University of Agricultural Sciences, 75007 Uppsala, Sweden. ${ }^{3}$ Department of Veterinary Integrative Biosciences, Texas A\&M University, College Station, TX 77843, USA. 'Department of Animal Breeding and Genetics, Ain Shams University, Shoubra El-Kheima, Cairo 11241, Egypt. ${ }^{5}$ Division of Immunology and Rheumatology, Department of Medicine, Stanford University, Stanford, CA 94305, USA. ${ }^{\square}$ email: leif.andersson@imbim.uu.se; syounis@stanford.edu
} 
${ }^{10}$. Moreover, miR483 can self-regulate its own expression by indirectlyenhancing the expression of USF1, which in turn stabilizes the transcriptional complex on the miR483 locus ${ }^{14}$. miR483-3p was found to target DLC-1, BRCA-1 and PUMA which are a candidate tumor repressor, a tumor repressor and an apoptosis modulator, respectively ${ }^{11,15,16}$. On the other hand, miR483-5p was found to increase tumorigenesis ${ }^{17}$ and to be an accurate marker to categorize certain tumors as benign or malignant ${ }^{18}$. Interestingly, it has been reported that miR483-5p binds directly to the 5 'UTR region of $I g f 2$ and thereby enhances $\operatorname{Igf} 2$ transcription ${ }^{17}$.

Here, we explore the functional significance of ZBED6 by a miRNA-seq screen using our Zbed6-KO and Igf2-KI mice to assess whether ZBED6 regulates miRNA in general and miR483 in particular. Furthermore, we investigate the role of $m i R 483$ in regulating Igf2 expression by knocking it out from Igf2 $2^{+/+}$and $I g f 2^{\mathrm{dGGCT}} \mathrm{C} 2 \mathrm{C} 12$ mouse myoblasts.

\section{Materials and methods}

Animals and tissues. In the current study we used a mouse model that lacks ZBED6 expression (KO) and another mouse model carrying a single base change ( $\mathrm{G}$ to $\mathrm{A}$ ) disrupting the ZBED6 binding site at the paternal allele of $\operatorname{Ig} f 2^{p A / m G}(\mathrm{p}=\text { paternal, } \mathrm{m}=\text { maternal })^{9}$. The pedigree based on mating male $Z b e d 6-\mathrm{KO} / \mathrm{WT}, \operatorname{Igf} 2^{\mathrm{pA} / m G}$ and female Zbed6-KO/WT, Igf2 $2^{G / G}$ mice was established previously ${ }^{9}$. From this cross, Zbed6-WT/WT, Igf2 ${ }^{G / G}$ (referred to as WT), Zbed6-KO/KO, Igf2 $2^{G / G}$ (referred to as Zbed6-KO) and Zbed6-WT/WT, Igf2 ${ }^{p A / m G}$ (referred to as Igf2-KI) mice were generated. WT, Zbed6-KO and Igf2-KI litter mates were anesthetized at the age of 20 weeks by injecting $16 \mu \mathrm{L} / \mathrm{g}$ body weight with $2.5 \%$ Avertin (2,2,2-tribromo ethanol and tertiary amyl alcohol). Skeletal muscle, kidney and liver tissues were collected and saved in RNA-later (Sigma, St. Louis, Missouri, United States).

Mice were kept at Uppsala University and Karolinska Institute. The experimental work was approved by the Uppsala Ethical Committee on Animal Research (\#C63/15 and \#C143/15) and the Stockholm Ethical Committee (\#N38/15). The rules of the Swedish Animal Welfare Agency were followed, and were in compliance with the European Communities Council Directive of 22 September 2010 (2010/63/EU). The study was carried out in compliance with ARRIVE guidelines.

Total RNA extraction from mouse tissues and miRNA-seq. Total RNA including miRNA were extracted from skeletal muscle, kidney and liver from WT, Igf2-KI and Zbed6-KO mice. Extractions were done using the miRNeasy mini kit (Qiagen, Hilden, Germany). Quality control and estimates of concentration of the extracted RNA samples were done using TapeStation 2200 (Agilent Technologies, Santa Clara, California, United States) and a NanoDrop ND-1000 spectrophotometer (Thermo Fisher Scientific, Waltham, Massachusetts, United States). miRNA sequencing was done (Center for Genomic Regulation, Barcelona, Spain) for three samples per tissue for each of the three genotypes (total of 27 samples) on a HiSeq system (Illumina, San Diego, California, United States) generating 8 to 16 million 50-bp single-end reads per sample.

Cell culture, Igf $2^{\mathrm{dGGCT}}$ and $/ g f 2^{+/+} \mathrm{C} 2 \mathrm{C} 12$ myoblasts. $I g f 2^{\mathrm{dGGCT}}$ mouse $\mathrm{C} 2 \mathrm{C} 12$ myoblast cells were previously generated using CRISPR/Cas9 and involved a deletion of the nucleotides "GGCT" from the intronic ZBED6 binding site in $I g f 2^{19}$. The $I g f 2^{\mathrm{dGGCT}}$ and $I g f 2^{+/+}$cells were cultured in Dulbecco's Modified Eagle Medium (DMEM) complemented with $10 \%$ heat-inactivated fetal bovine serum, penicillin $(0.2 \mathrm{U} / \mathrm{mL})$, streptomycin $(0.2 \mu \mathrm{g} / \mathrm{mL})$ and L-glutamine $(0.2 \mu \mathrm{g} / \mathrm{mL})$ (Gibco, Waltham, Massachusetts, United States). Cells were incubated at $37{ }^{\circ} \mathrm{C}$ and $5 \% \mathrm{CO}_{2}$ and were split upon reaching $80-90 \%$ confluency. Cells were tested for mycoplasma infection using MycoAlert mycoplasma detection kit (Lonza, Basel, Switzerland) and were shown to be free of infection.

Generation of $\mathbf{m i R 4 8 3 ^ { - I - }}$ cells using CRISPR. Guide RNA sequences targeting the mouse miR483 gene were designed using CRISPRdirect ${ }^{20}$. Two guide-RNA sequences with scores above 90 were chosen. Four single-stranded DNA oligos (Supplementary Table 1) were synthesized (IDT-DNA, Coralville, Iowa, United States), annealed by mixing the forward and reverse oligonuclotides in a $1: 1$ ratio, heated to $95{ }^{\circ} \mathrm{C}$ and allowed to cool down to room temperature at a speed of $0.5^{\circ} \mathrm{C} / \mathrm{min}$. Annealed oligonuclotides were cloned into the pSpCas9(BB)-2A-GFP (PX458) plasmid (Addgene) to obtain the two miR483-gRNA-plasmid constructs.

$I g f 2^{+/+}$and $I g f 2^{\mathrm{dGGCT}} \mathrm{C} 2 \mathrm{C} 12$ cells $^{19}$ were seeded and incubated for $24 \mathrm{~h}$ prior to transfections. Transfections were done when the cells reached 80-90\% confluency using JetPRIME transfection reagent (Polyplus transfection, BIOPARC, Illkirch-Graffenstaden, France). The constructs were transfected along with a linear puromycin marker (Clontech, Mountain View, California, United States) and the selection of stable cells was done by adding $1 \mu \mathrm{g} / \mathrm{mL}$ puromycin (Sigma) for 2 weeks. Cell culture media were changed every day during the first 3 days, and then whenever needed during the 2 weeks period. Control cells were obtained by following the same procedure but transfecting C2C12 cells with pSpCas9(BB)-2A-GFP (PX458) plasmid lacking any gRNA.

miR483 mimics and inhibitors. The generated $m i R 483^{-/-}$cells and miR483 ${ }^{+/+}$control cells were seeded and transfected as described above. $m i R 483^{-1-}$ cells were transfected with miR483-3p and miR483-5p mimics. Whereas miR483 ${ }^{+/+}$control cells were transfected with miR483-3p and miR483-5p inhibitors.

DNA and RNA extraction. DNA was extracted using the DNeasy blood and tissue kit (Qiagen) and the concentration was measured using a NanoDrop ND-1000 spectrophotometer (Thermo Fisher Scientific). Total RNA was extracted using the RNeasy mini kit (Qiagen). Total RNA including miRNA was extracted using the 
miRNeasy mini kit (Qiagen). RNA concentration and quality were measured using Tape Station 2200 (Agilent Technologies). All RNA extractions were combined with DNase I (Qiagen) treatment.

RT-qPCR. Total RNA and miRNA were converted to cDNA using the high capacity reverse transcription kit (Applied Biosystems, Foster City, California, United States), and the TaqMan advanced cDNA synthesis kit (Applied Biosystems), respectively. cDNA was used to perform quantitative PCR (qPCR) on a QuantStudio 6 Flex instrument using the QuantStudio Real-Time PCR software v1.3 (Applied Biosystems). The volume of the qPCR reactions was set to $10 \mu \mathrm{L}$.

Proliferation assay. $\mathrm{C} 2 \mathrm{C} 12$ cells with different genotypes were cultured in 6-well plates. Each cell clone was cultured in 16 wells $\left(10^{4}\right.$ cells/well) at day 0 . Then at each day, for a total of 8 days, the number of cells were counted from two wells for each of the clones. Cell counting was performed by washing the cells with PBS, adding $0.5 \mathrm{~mL}$ trypsin for $3 \mathrm{~min}$ at RT before adding $1 \mathrm{~mL}$ of cell culture medium, which includes FBS, to deactivate trypsin. A volume of $12.5 \mu \mathrm{L}$ of the cell mixture was mixed in a 1:1 ratio with Trypan blue, and counted on a Countess II Automated cell counter instrument (Invitrogen).

RNA-Seq of cell lines. Total RNA was extracted from the developed stable cells as described above. The mRNA was selected using the Dynabeads mRNA DIRECT purification kit (Thermo Fisher Scientific). RNA-seq libraries were prepared using the SENSE Total RNA-Seq Library Prep kit (Lexogen, Campus-Vienna-Biocenter 5, Wien, Austria) and the libraries were amplified by a 17-cycle PCR. The quality and concentration of the prepared libraries were evaluated using TapeStation 2200 (Agilent Technologies). Equimolar amounts from each library were pooled and sent for sequencing at the SNP\&SEQ platform (Uppsala University, Uppsala, Sweden) using the NovaSeq 6000 system (Illumina). A total of eight libraries, comprising one library per clone for

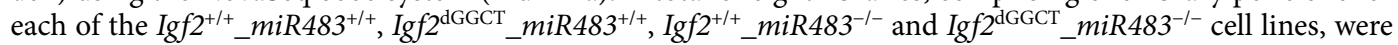
sequenced which generated 35 to 50 million 50-bp paired-end reads per library.

Bioinformatic analysis. Sequence reads were mapped to the reference mouse genome (mm10) using STAR 2.5.1b with default parameters ${ }^{21}$. HTSeq-0.6.1 (Python Package) ${ }^{22}$ was used to generate read counts. edgeR (Bioconductor package $)^{23}$ was used to identify differentially expressed (DE) genes using gene models and an annotation GTF file from the mouse genome assembly mm10 (downloaded from UCSC genome browser, http://www. genome.ucsc.edu). The abundance of gene expression was calculated as count-per-million (CPM) reads. Genes with less than one CPM in at least two samples were filtered out. The filtered libraries were normalized using the trimmed mean of M-values (TMM) normalization method ${ }^{24} . P$-values were corrected for multiple testing using the False Discovery Rate (FDR) approach. For miRNA samples, one of the WT muscle samples was shown to be an outlier during the analysis and was therefore excluded. The miRNA annotation information was obtained from miRbase (downloaded 2018-03-05).

A gene ontology analysis of the DE genes were conducted using the Clusterprofiler R package $\mathrm{e}^{25}$. All expressed genes were used as background, and the Biological Process and KEGG pathway tables were used to identify enriched GO terms. The gene set enrichment analysis (GSEA) was performed using the fgsea R package ${ }^{26}$. The genes were ranked based on the fold-change and the datasets were downloaded from the GSEA website (https:// www.gsea-msigdb.org/gsea/downloads.jsp). The RNA-seq reads have been submitted to the sequence read archive (http://www.ncbi.nlm.nih.gov/sra) with the Bioproject accession number PRJNA744495.

\section{Results}

miRNA-seq from Zbed6-KO, Igf2-KI and wild-type mice. To investigate whether the ZBED6 transcription factor controls miRNA expression, a miRNA-seq screen was performed using kidney, liver and skeletal muscle tissues from Zbed6-KO and WT C57BL/6 litter mates. Furthermore, because Igf2 appears to be the most important downstream target for ZBED6 ${ }^{9}$, we also performed a screen using our Igf2-KI mice in which the ZBED6 binding site in Igf2 is disrupted. This experimental design allows us to dissect if an altered expression of a miRNA in Zbed6-KO mice is mediated through the interaction with its binding site in Igf2 (Figs. 1 and S1).

The results show that ZBED6 has a minimal impact on regulating the expression of miRNAs in the tested tissues, with the exception of miR483-3p and miR483-5p that were upregulated in muscle and kidney tissue of Zbed6-KO mice (Figs. 1a-d and S1). A similar result was found in the Igf2-KI mice where miR483-3p and miR483-5p were significantly upregulated but only in muscle (Figs. 1a-d and S1) and another miRNA, miR3105$3 p$, showed upregulated expression in kidney (Figure S1). The up-regulation of miR483-3p and miR483-5p were validated by qPCR analysis (Fig. 1e, left). In liver, no significant differential expression was detected for any miRNA in any genotype comparison (Figure S1). In fact, neither miR483-3p nor miR483-5p were expressed at detectable levels (at least one read per million reads in at least two samples) in liver (Supplementary Table 2). qRT-PCR analysis confirmed the striking difference between muscle and liver tissue as regards the induction of Igf2 mRNA expression when the ZBED6-Igf2 axis is disrupted (Fig. 1e, right). RNAseq analysis assessing the expression of the three different Igf2 transcripts further documents the dramatic upregulation of Igf2 expression in skeletal muscle, heart and kidney in contrast to the minute upregulation in liver (Figure S2).

In conclusion, ZBED6 does not regulate miRNA expression in general, but ZBED6 inactivation or the disruption of its interaction with the Igf2 locus results in a major upregulation of miR483 expression in some tissues including skeletal muscle but not in liver. 
a
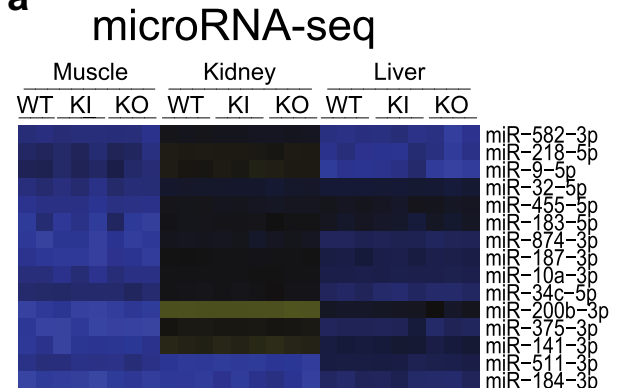

$\mathrm{m} R-32-5 \mathrm{p}$
$\mathrm{m} R \mathrm{R}-455-5 \mathrm{p}$
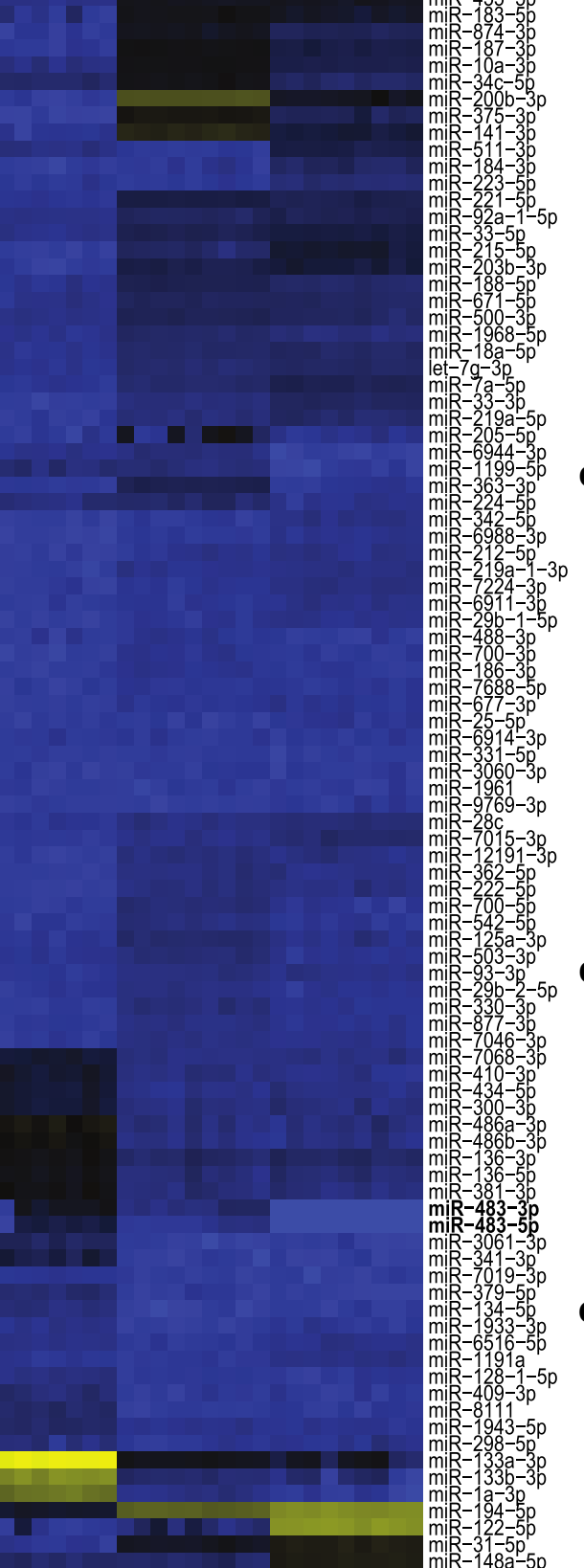

miR-148a-5p
miR-1247-5p

miR-1247-5p
miR-335-3p

Low

High

b

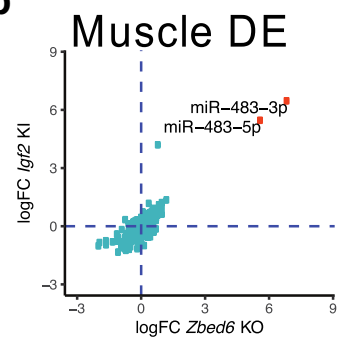

Liver DE

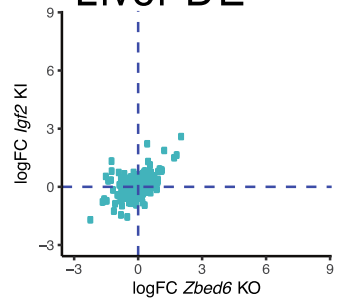

c $\quad \operatorname{miR} 483-3 p$

$\operatorname{miR} 483-5 p$

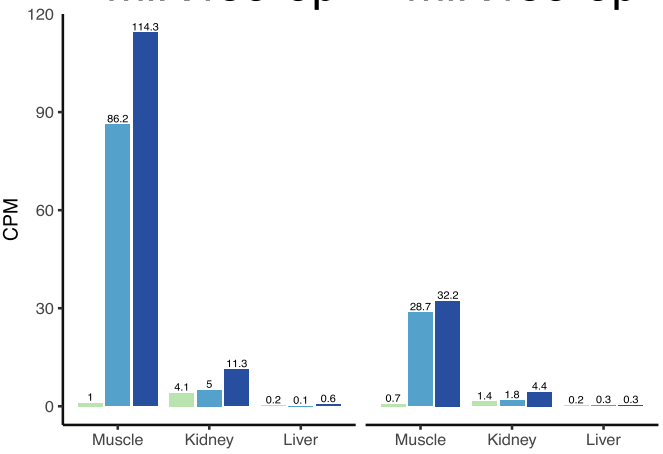

d Muscle

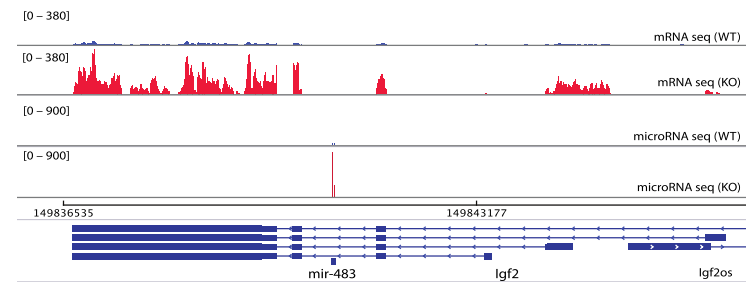

\section{e qPCR analyses}
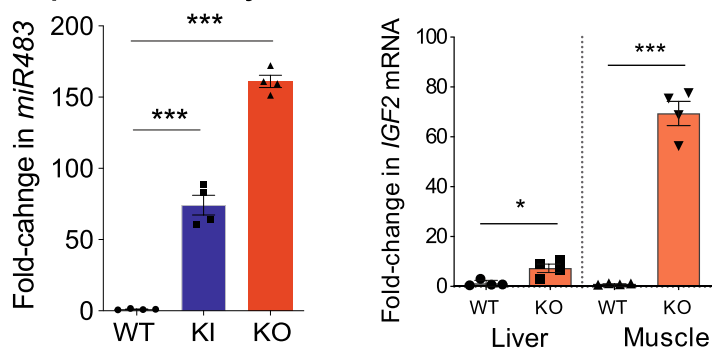

Figure 1. miRNA-seq analysis of mouse tissues in wild-type, Zbed6-KO and Igf2-KI mice. (a) Heatmap showing 100 expressed miRNAs across genotypes analyzed in each tissue separately, with miR483-3p and miR483-5p indicated in bold. (b) Scatterplots of log fold-change of miRNAs in Zbed6-KO and Igf2-KI mice compared with wild-type in muscle, kidney and liver tissues. (c) Expression of miR483-3p and miR483-5p in muscle, kidney and liver. The bars represent expression in counts per millions (CPM). (d) Uniquely mapped mRNA and miRNA reads at the Igf2 locus in wild-type and Zbed6-KO muscle tissue. (e) qPCR analysis showing the expression levels of miR483 in muscle tissue of WT, Igf2-KI and Zbed6-KO mice (left) and of Igf2 mRNA in liver and muscle of Zbed6-KO mice (right). Graph shows the fold changes (mean \pm SEM) compared to WT. ${ }^{* *}$ corresponds to $P<0.001$ in a two-tailed Student's t-test. 
Expression levels of $\operatorname{lgf} 2$ and $m i R 483$ in $\lg f 2^{\mathrm{dGGCT}}$ and $\operatorname{lgf2^{+/+}} \mathrm{C} 2 \mathrm{C} 12$ cells. We previously established an Igf2 ${ }^{\mathrm{dGGCT}} \mathrm{C} 2 \mathrm{C} 12$ myoblast cell line using CRISPR ${ }^{19}$ which resulted in a deletion of four base pairs (GGCT) of the ZBED6 binding site in an intron of Igf2 (Fig. 2a). Since miR483 expression was upregulated in skeletal muscles of both Zbed6-KO and Igf2-KI mice (Fig. 1b,c), which both show more than 20 -fold upregulated Igf2 expression at the mRNA level in skeletal muscle ${ }^{9}$, a qPCR experiment was performed to test if the expression of $m i R 483$ is also higher in $I g f 2^{\mathrm{dGGCT}}$ compared to $I g f 2^{+++} \mathrm{C} 2 \mathrm{C} 12$ cells. In fact, the qPCR results showed a 12 -fold increase of miR483-3p expression and more than two-fold increase in miR483-5p expression in the Igf2 ${ }^{\mathrm{dGGCT}}$ cells (Fig. 2b). Furthermore, Igf2 showed a 40 -fold increase in mRNA expression in the Igf $2^{\mathrm{dGGCT}}$ cells compared to Igf2 $2^{++}$cells (Fig. 2c). Thus, the consequences of disruption of the ZBED6-Igf2 interaction in C2C12 cells mimic the changes in Igf2 and miR483 expression in skeletal muscle in transgenic mice.

Generation of miR483-1- $\mathrm{C} 2 \mathrm{C} 12$ cells. In order to further investigate the role of miR483 in regulating Igf2 expression in $\mathrm{C} 2 \mathrm{C} 12$ cells, a guide-RNA was designed to target the miR483 gene using the CRISPR-Cas9 method $^{27,28}$. The plasmid was transfected into Igf2 ${ }^{+/+}$and $\operatorname{Igf2} 2^{\mathrm{dGGCT}} \mathrm{C} 2 \mathrm{C} 12$ cells in an attempt to knock out miR483 (Fig. 2a). Two miR483 $3^{-l-}$ clones each were generated from Igf2 $2^{+/+}$and Igf $2^{\mathrm{dGGCT}} \mathrm{C} 2 \mathrm{C} 12$ cells.

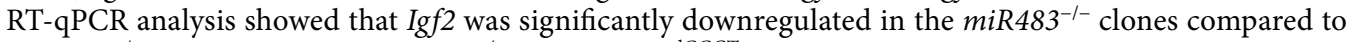

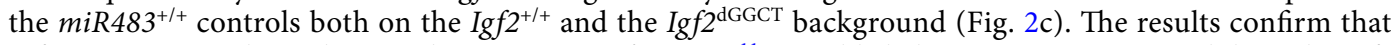
Igf2 expression is dependent on the expression of $m i R 483^{11}$ most likely because miR483-5p stabilizes the Igf2 transcripts $^{17}$.

miR483 inhibitors and mimics. Igf2 expression was then analyzed in Igf2 $2^{+/+}$and Igf2 ${ }^{\mathrm{dGGCT}} \mathrm{C} 2 \mathrm{C} 12$ cells that were transiently transfected with miR483-3p and miR483-5p oligonucleotide inhibitors. Transient transfection of miR483-5p inhibitors caused a significant downregulation of Igf2 expression compared to un-transfected cells and mock controls (Fig. 2d,e). Furthermore, the miR483 $3^{--}$clones were transfected with miR483-3p and miR483-5p mimics which resulted in a significant upregulation of Igf2 expression in particular using miR483-5p (Fig. 2f,g), confirming the important role of miR483-5p for controlling Igf2 expression in these cells.

Disruption of miR483 expression affects the proliferation of $\mathrm{C} 2 \mathrm{C} 12$ cells. In order to evaluate the phenotypic effects of miR483 downregulation, we measured the proliferation rate of the $m i R 483^{-1-} \mathrm{C} 2 \mathrm{C} 12$ in $I g f 2^{+/+}$and Igf $2^{\mathrm{dGGCT}}$ cells where the ZBED6-Igf2 axis is disrupted. In comparison with the Igf $\mathrm{d}^{\mathrm{dGGCT}}{ }_{-} m i R 483^{+/+}$ cells, the knocking out of $m i R 483$ from the $I g f 2^{+/+}$and $I g f 2^{\mathrm{dGGCT}}$ cells resulted a modest but statistically significant reduction in cell proliferation starting at days 3 and 5, respectively. Whereas, the $\operatorname{Ig} f^{+/+}$cells in which miR483 was not knocked out showed a statistically significant reduction in cell proliferation only at day 8 (Fig. $2 \mathrm{~h}$ ). The result is in agreement with the altered expression of Igf2 and miR483 in the mutant cells as explained above (Fig. 2a-g), and shows that the marked upregulation of Igf2 expression due to disruption of the ZBED6-Igf2 axis in this cell line is dependent on the expression of miR483. (Fig. 2h).

RNA-seqanalysis. An RNA-seq analysiswasperformed using the $I g f 2^{+/+}{ }_{2} m i R 483^{-/}$and $I g f 2^{\mathrm{dGGCT}}$ $m i R 483^{-/-}$

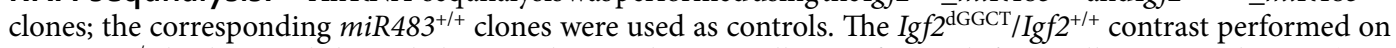
a $m i R 483^{+/+}$background showed almost a thousand statistically significant, differentially expressed genes (Figure S3, Supplementary File 2) with Igf2 being one of the genes with the highest fold-change (Fig. 3a,b). Sec-

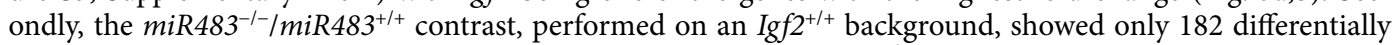
expressed genes with almost half of the genes being shared with the Igf2 $\mathrm{dGGCT}^{\mathrm{d}} / \mathrm{Igf} 2^{+/+}$contrast (Figs. 3c,d and S3). Moreover, the $m i R 483^{-/-} / m i R 483^{+/+}$contrast, performed on an Igf2 $2^{\mathrm{dGGCT}}$ background, showed only 164 differentially expressed genes with $76 \%$ of the genes shared with the Igf $2^{\mathrm{dGGCT}} / \mathrm{Ig} f^{+/+}$contrast (Figs. 3e,f and S3). The

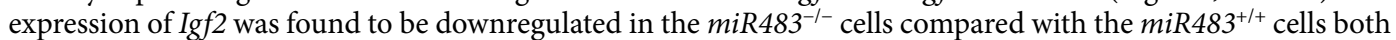
on the Igf2 $2^{+/+}$and the $\operatorname{Ig} f^{\mathrm{dGGCT}}$ background (Fig. 3c-f) in agreement with the qPCR analysis (Fig. 2c).

To investigate whether genes that are differentially expressed upon knocking-out miR483 (background: $I g f 2^{+/+}$), are also differentially expressed in the Igf $2^{\mathrm{dGGCT}} / \mathrm{Ig} f^{2++}$ contrast (background: $m i R 483^{+/+}$), a scatter plot was made comparing the outcome of the two contrasts (Figure S4A). Out of the 182 differentially expressed genes that are significant in the $m i R 483^{-/-} / m i R 483^{+/+}$contrast, 96 are significant in this contrast, but not in the $\operatorname{Igf} 2^{\mathrm{dGGCT}} / \mathrm{Ig} \mathrm{2}^{+/+}$contrast. However, almost half of these 96 genes (43 genes) showed a $\log _{2}$ fold-change greater

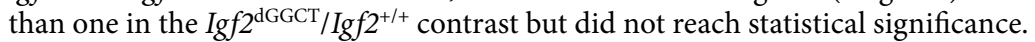

Similarly, the differential gene expression in the Igf $2^{\mathrm{dGGCT}} / \operatorname{Igf} 2^{+/+}$contrast (background: $m i R 483^{+/+}$) and the

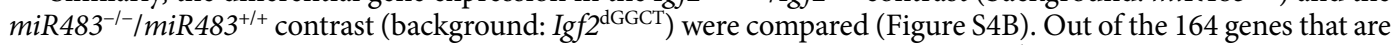
significant in the $m i R 483^{-/-} / \mathrm{miR} 483^{+/+}$contrast, 40 were not significant in the Igf $2^{\mathrm{dGGCT}} / \mathrm{Igf2} 2^{+/+}$contrast although more than half of the genes ( 24 genes) showed a $\log _{2}$ fold-change value greater than 1 in the latter contrast.

These comparisons demonstrate that the 130 differentially expressed genes reaching statistical significance only in the two $\mathrm{miR} 483^{-/-} / \mathrm{miR} 483^{+/+}$contrasts, contained 67 genes (49\%) with at least a two-fold change in gene expression in the Igf $2^{\mathrm{dGGCT}} / \operatorname{Ig} f^{+/+}$contrast, but the data did not reach statistical significance probably due to a limited statistical power. Moreover, the expression of 64 out of these 67 genes showed an opposite trend between $I g f 2^{\mathrm{dGGCT}}$ cells, where Igf2 expression is up-regulated, and $m i R 483^{-I-}$ cells, where Igf2 expression is down-regulated.

In conclusion, the considerable overlap in the genes showing differential expression in the $\operatorname{Igf} 2^{\mathrm{dGGCT}} / \operatorname{Igf} 2^{+/+}$ contrast and in the two $m i R 483^{-/-} / m i R 483^{+/+}$contrasts implies that an important biological function for miR483 in these myoblast cells is its regulation of Igf2 expression. 
a

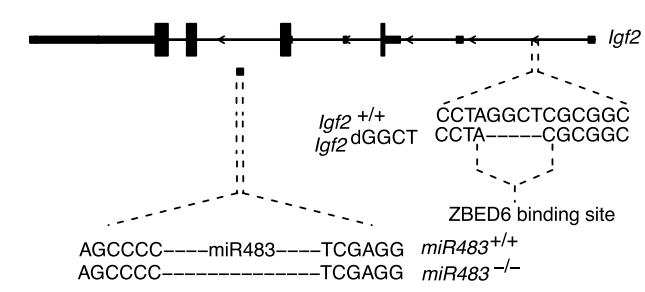

C

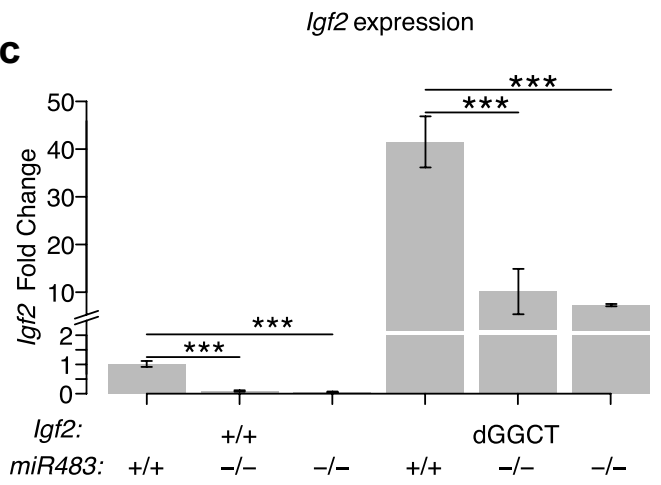

e

$$
\operatorname{lgf2}^{\mathrm{dGGCT}} \text { miR483 }^{+/+}
$$
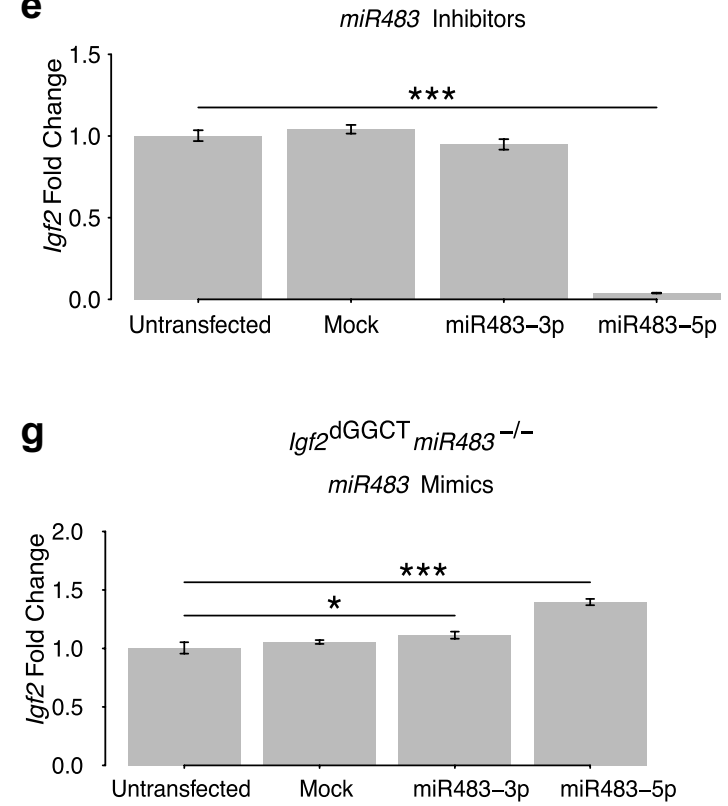

b

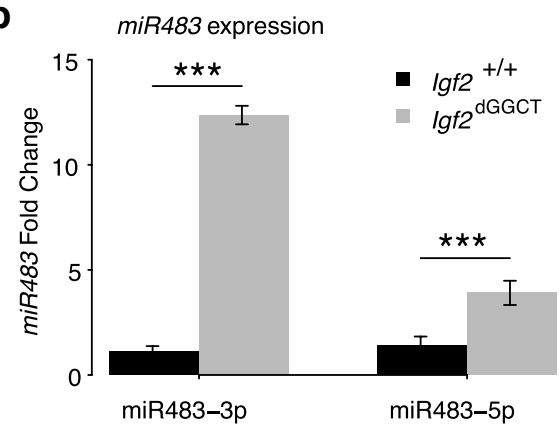

d
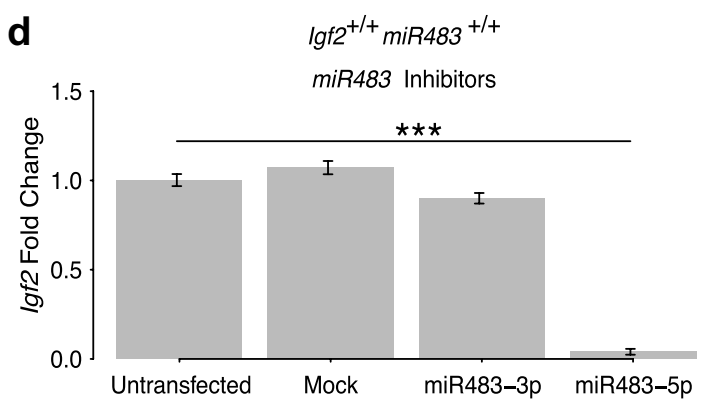

$\mathbf{f}$

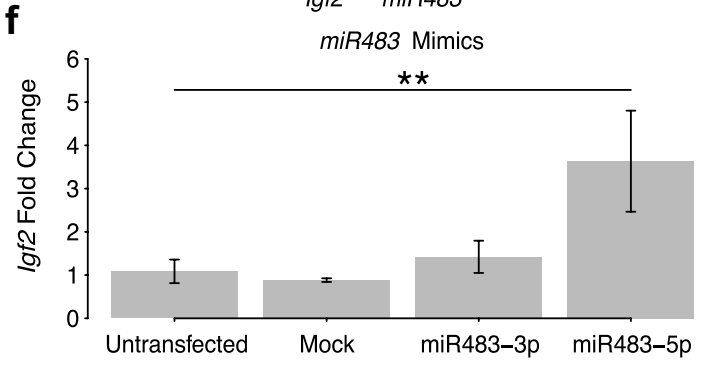

h

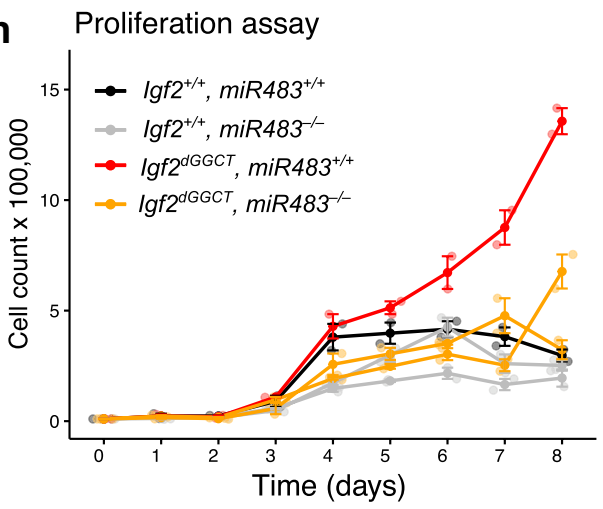

Figure 2. Disruption of miR483 and its effect on Igf2 expression in mouse C2C12 myoblast cells. (a) A depiction of the Igf2 locus showing the Igf $2^{\mathrm{dGGCT}}$ mutation at the ZBED6 binding site, and the miR483 sequences that were removed by CRISPR/Cas9. (b) qPCR analysis showing the expression levels of miR483

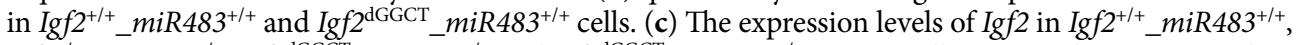

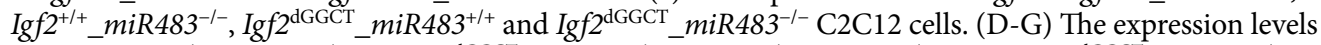

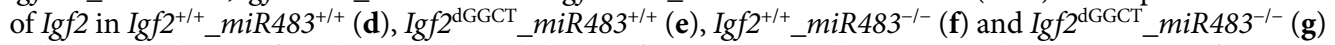
cells transiently transfected with either inhibitors of miR483-3p and miR483-5p (d,e) or mimics of miR483-3p and miR483-5p (f,g). The ddCt method was used to normalize miR483 against miR15b-5p and Igf2 against 18 s rRNA. ${ }^{*},{ }^{* *}$ and ${ }^{* * *}$ corresponds to $P<0.05,0.01$ and 0.001 in a two-tailed t-test, respectively. (h) Cell proliferation of wild-type and mutant $\mathrm{C} 2 \mathrm{C} 12$ cells. Single clones were used for $I g f 2^{+/+}, m i R 483^{+/+}$and $\operatorname{Igf} 2^{d G G C T}$, $m i R 483^{+/+}$, while two clones each were used for $I g f 2^{+/+}, m i R 483^{-/-}$and $I g f 2^{d G G C T}, m i R 483^{-/-}$. Each of the six clones was run in duplicates. 


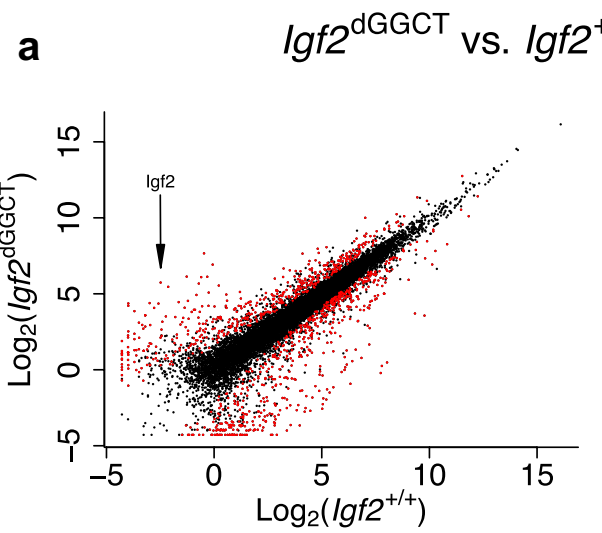

, Background: $m i R 483^{+/+}$
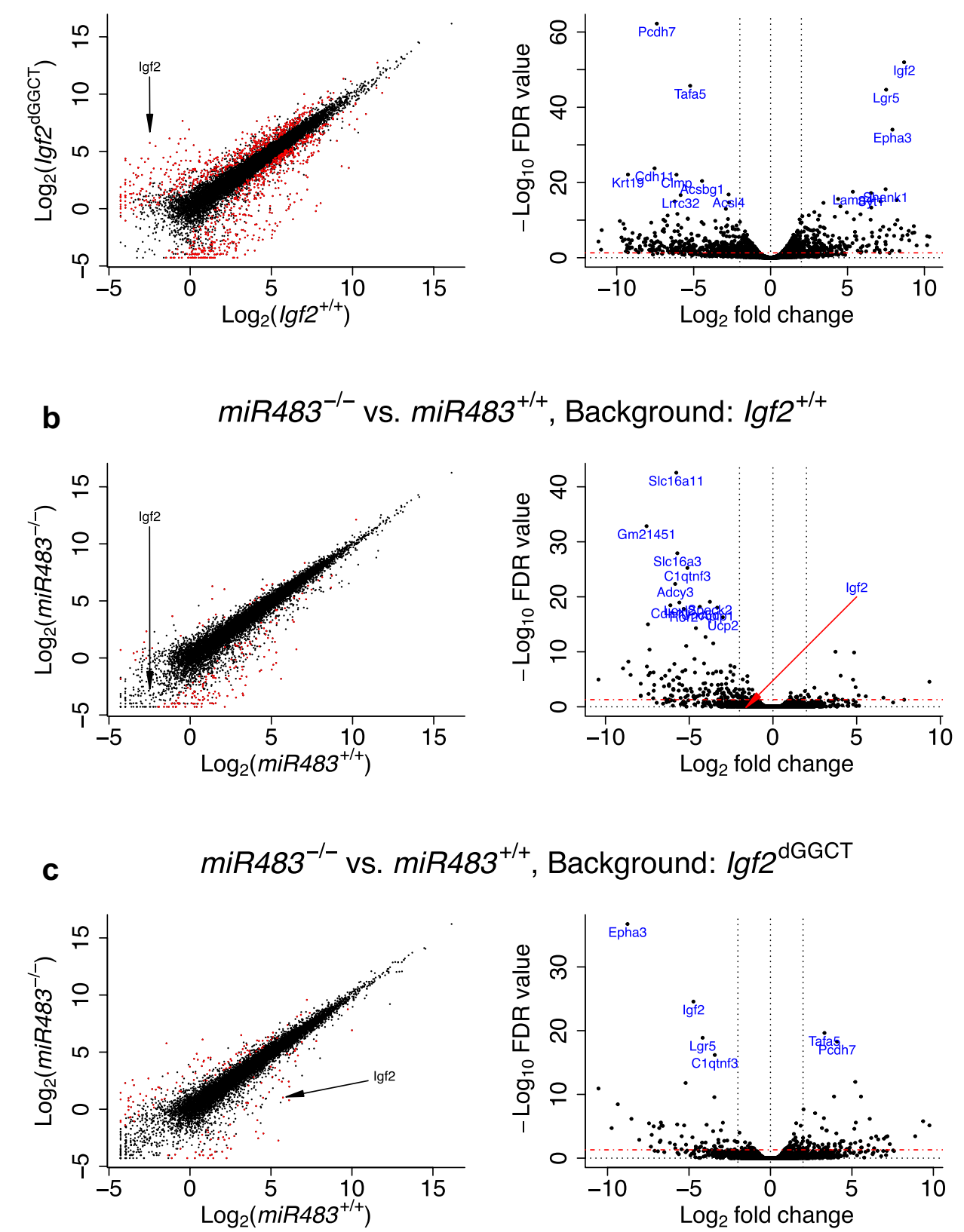

$3^{+/+}$, Background: Igf2 ${ }^{\mathrm{dGGCT}}$

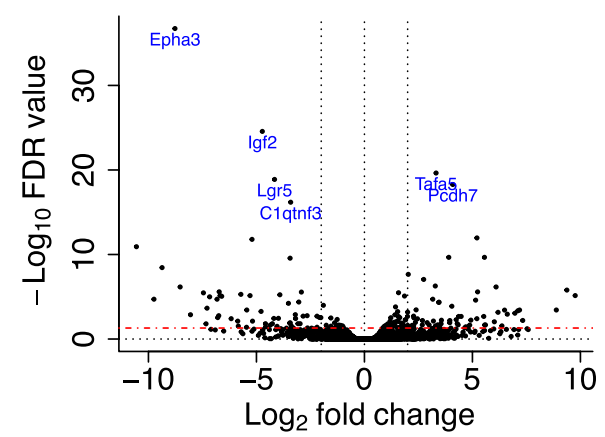

Figure 3. Results of RNA-seq analysis comparing $\operatorname{Igf2} 2^{+/+}, \operatorname{Igf2} 2^{\mathrm{dGGCT}}, \mathrm{miR} 483^{+/+}$and $m i R 483^{-/-} \mathrm{C} 2 \mathrm{C} 12$ cells. Scatter plots of RNA-seq expression levels in the $\log _{2}$ scale between the different contrasts (left), and volcano plots showing the $\log _{2}$ fold-change and the $-\log _{10}$ FDR values (right). Expression values are the averages of two samples per cell type. Statistically significant differentially expressed genes with $\log _{2}$ FC $>2$ and $-\log _{10}$ FDR $>1.3$ are plotted in red in the scatter plots, and $-\log _{10} \mathrm{FDR}>1.3$ are indicated by a horizontal dashed red line in the volcano plots.

Pathway analysis of $\mathrm{miR}_{\mathbf{4}} \mathbf{8 3 ^ { - / - }}$ cells. In order to further explore the function of $m i R 483$, we performed gene set enrichment analysis (GSEA) and gene ontology (GO) analysis using the differentially expressed (DE) genes in $m i R 483^{-l-}$ cells. The GSEA of ranked DE genes in $m i R 483^{-1-}$ cells revealed a significant enrichment of myogenesis among the down-regulated genes, and a significant enrichment of genes involved in the P53 pathway among up-regulated genes (Fig. 4a, top). The heatmaps present the expression of the subset of genes that contributed the most to the enrichment signals, including Igf2, Myog, Myh3 and Tnnt2 as key down-regulated genes with a role in myogenesis and Cdkn1a, Steap 3 and Tob1 as key upregulated genes in the P53 pathway (Fig. 4a, 


\section{a GSEA: Hallmarks of miR483-1-}
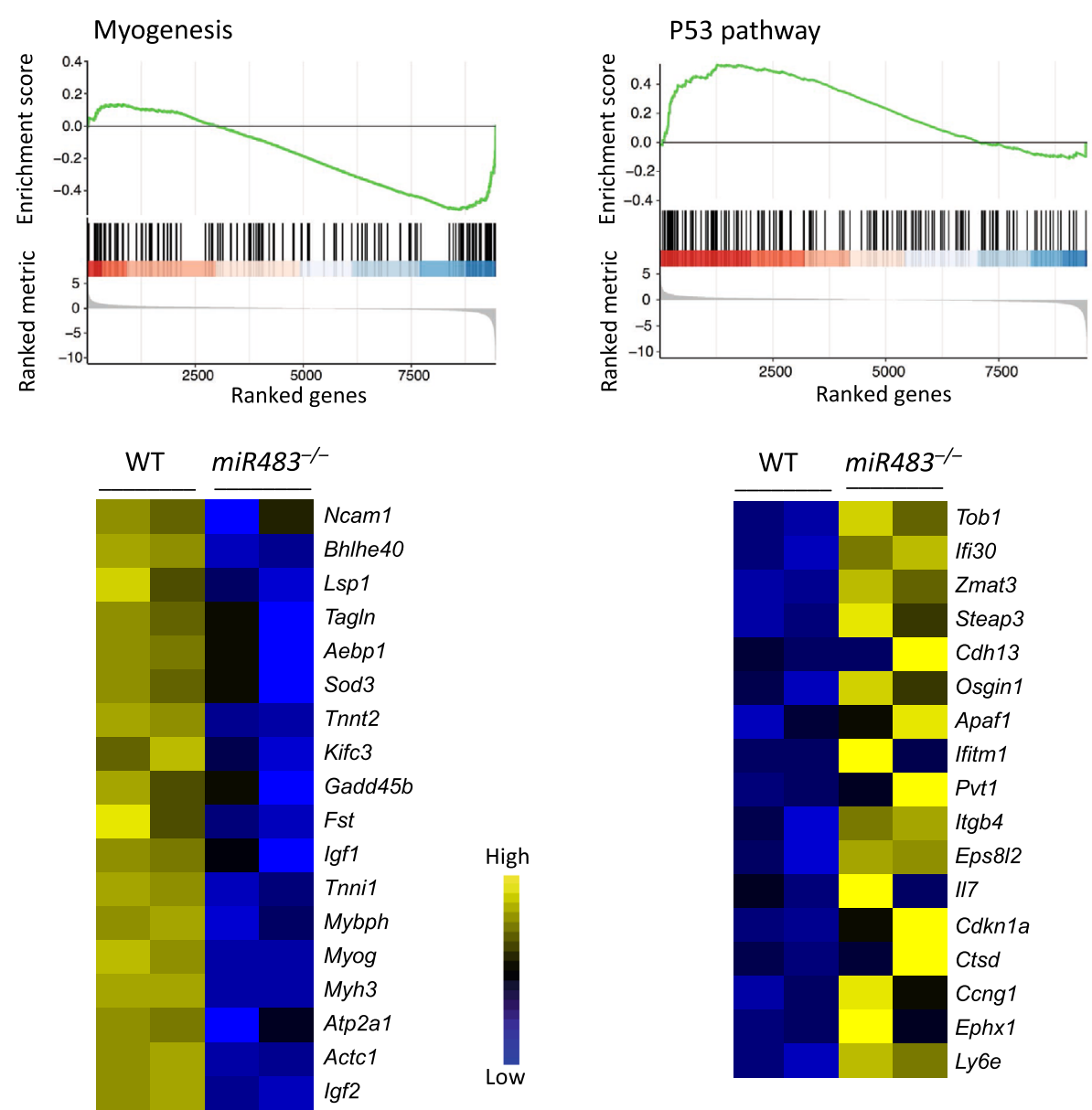

\section{b GO analysis of DE genes (FDR $<0.05)$ in miR483-- $^{-1}$

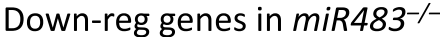 \\ Wnt pathway genes}

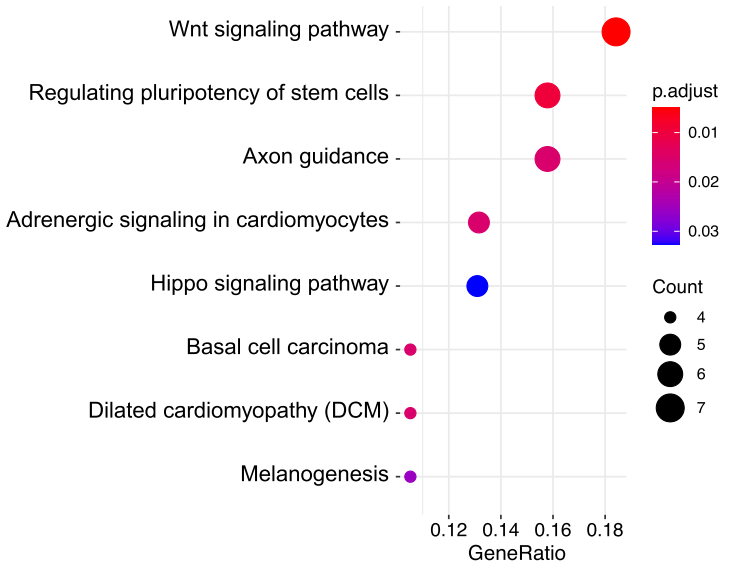

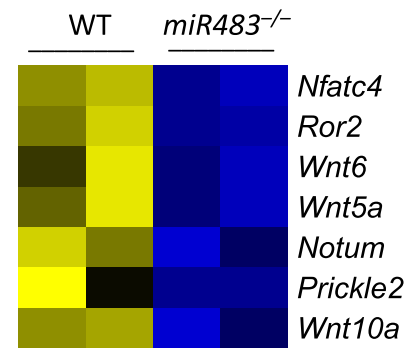

Figure 4. Pathway analysis of RNA-seq data comparing $m i R 483^{+/+}$and $m i R 483^{-/-} \mathrm{C} 2 \mathrm{C} 12$ cells on an $\mathrm{Igf2} 2^{+/+}$ background. (a) Gene set enrichment analysis of ranked DE genes in miR483 ${ }^{-1}$ cells using hallmark gene sets (top) and heatmap of the genes contributing to the myogenesis or P53 pathway (below). (b) KEGG pathway analysis of down-regulated genes in $m i R 483^{-/-}$cells and heatmap of Wnt pathway-related genes. 
below). Furthermore, the GO analysis of significantly down-regulated genes (FDR $<0.05)$ in $m i R 483^{-1-}$ cells showed a significant enrichment for the Wnt signaling pathway (Fig. 4b).

The interaction between miR483 and the ZBED6-Igf2 axis. To gain further insight into the impact of $m i R 483$ on the ZBED6-Igf2 axis, we characterized the expression pattern in Igf2 ${ }^{\mathrm{dGGCT}}$ cells after knocking out miR483. Hierarchical cluster analysis of mutant $\mathrm{C} 2 \mathrm{C} 12$ cells shows distinct differences between genotypes, with largest distance between $I g f 2^{+/+}$and Igf $2^{\mathrm{dGGCT}}$ groups. Knocking out $m i R 483$ from Igf2 ${ }^{\mathrm{dGGCT}}$ cells $\left(\mathrm{dKO}^{\text {mir483/ }}\right.$ dGGCT) caused transcriptional changes resulting in an intermediate position of these cells in a cluster analysis including Igf $2^{+/+}$and Igf $2^{\mathrm{dGGCT}}$ cells (Fig. 5a). In agreement with this hierarchical clustering, the expression of Igf2 was highest in Igf2 $2^{\mathrm{dGGCT}}$ cells, where the ZBED6-Igf2 axis is disrupted, and moderate in $\mathrm{dKO}^{\text {mir483/dGGCT }}$ cells (Fig. 5b). The KEGG pathway analysis of DE genes in Igf $2^{\mathrm{dGGCT}}$ cells revealed a significant enrichment of the PI3K-Akt signaling pathway among the up-regulated genes (Fig. $5 c$,d). Remarkably, the opposite pattern was observed in $\mathrm{dKO}^{\text {mir483/dGGCT }}$ cells, where $m i R 483$ is abolished, and the PI3K-Akt signaling pathway was enriched among the down-regulated genes (Fig. 5c,d). Taken together, these results further underscore the importance of an intact miR483 function for the full effect of disrupting the ZBED6-Igf2 interaction in C2C12 cells.

\section{Discussion}

The transcription factor ZBED6 is an innovation in placental mammals, and has evolved from a domesticated DNA transposon ${ }^{4}$. The extremely high sequence conservation $(\sim 100 \%)$ of the DNA-binding domain across species implies that it has an essential function in placental mammals ${ }^{4}$. A well-established function of ZBED6 is its regulation of Igf2 expression after birth which has a strong impact on muscle growth and the size of internal organs $^{3,9}$. The current study shows that it is not a regulator of miRNA expression in general but it suppresses the expression of miR483 at least in muscle cells, as revealed by enhanced expression in Zbed6-KO and Igf2-KI mice, and in Igf2 $2^{\mathrm{dGGCT}} \mathrm{C} 2 \mathrm{C} 12$ cells (Fig. 6). The data for kidney cells is mixed since both miR483-5p and miR483-3p were upregulated in Zbed6-KO mice but not in Igf2-KI mice. The expression of miR483-5p and miR483-3p in liver was unaltered in both mouse models (Supplementary Table 2). These results combined with the wellestablished association between up-regulated expression of miR483 and tumorigenesis ${ }^{11,12,16-18}$ implies that disruption of the ZBED6-Igf2 axis, either by impairing ZBED6 expression or blocking its access to the binding site in an intron of $I g f 2$, can promote tumor growth in those cell types where ZBED6 suppresses miR483 expression.

The analysis also revealed upregulated expression of miR3105-3p in kidney of Igf2-KI mice (Figure S1D), which is located at a distance of $0.5 \mathrm{Mb}$ from a ZBED6 binding site identified by ChiP-seq analysis in mouse $\mathrm{C} 2 \mathrm{C} 12$ cells $^{4}$ and only $1.4 \mathrm{Mb}$ from the Igf2 gene on mouse chromosome 7. Igf1r, Igf2r, Igf2 $b p 1$ and Igfbp4 are all predicted to be potential targets of miR3105-3 $\mathrm{p}^{29}$. But none of these predicted targets showed significant differential expression in RNA-seq analysis of mouse kidneys comparing Zbed6-KO and WT mice. However, this does not necessarily mean that the proteins of these genes are not differentially expressed, as miRNAs can mediate translational repression ${ }^{30}$.

The present study shows that $m i R 483$ is an important mediator for how ZBED6 regulates Igf2 expression. ZBED6 suppresses Igf2 expression partially by suppressing miR483 (Fig. 6). This is illustrated by the finding that disruption of the ZBED6 binding site in Igf $2^{\mathrm{dGGCT}} \mathrm{C} 2 \mathrm{C} 12$ cells results in a $\sim 40$-fold upregulation of Igf2 expression but this is reduced to an eightfold upregulation when miR483 is also deleted. Furthermore, our results show that miR483-5p, but not miR483-3p, enhances Igf2 expression in mouse C2C12 cells, and this is consistent with the original report of this mechanism using human tumor cells ${ }^{17}$.

The data on C2C12 cells as well as our miRNA-seq data showed that ZBED6 has a major effect on regulating $m i R 483$ expression in muscle cells, which in turn enhances the effect on Igf 2 expression. In contrast, Zbed6 inactivation had no impact on $m i R 483$ expression in liver (Figure S1E), a tissue where we did not detect $m i R 483$ expression at all. Interestingly, a previous study in pigs showed that inactivation of the ZBED6 binding site in Igf2 has a highly significant effect on Igf2 expression in skeletal and cardiac muscle but not in liver ${ }^{3,4}$. It is possible that low expression of $m i R 483$ in liver contributes to this lack of altered expression.

Our RNA-seq data comparing the transcriptome in $m i R 483^{+/+}$and $m i R 483^{-/-} \mathrm{C} 2 \mathrm{C} 12$ cells indicate that a major function of $m i R 483$ in this cell type is the regulation of $I g f 2$ expression because $\sim 50 \%$ of transcripts showing differential expression between $m i R 483^{+/+}$and $m i R 483^{-/-}$cells also showed differential expression between $I g f 2^{+/+}$and Igf2 $2^{\mathrm{dGGCT}}$ cells (Figure S3). This proportion was highest (76\%) when miR483 was knocked out on an Igf2 $2^{\mathrm{dGGCT}}$ background, where Igf2 and $m i R 483$ expression are enhanced. The pathway analysis of differentially expressed genes between $\mathrm{miR} 483^{-/-}$and $\mathrm{miR} 483^{+/+}$cells on an Igf2 $2^{+/+}$background showed a negative enrichment of myogenesis genes (Fig. 4a). This is in agreement with the known role of Igf2 in myogenesis ${ }^{19}$. Interestingly, we found that modulating the expression of miR483 is sufficient to alter the downstream signaling pathway of IGF2. For instance, PI3K-Akt signaling pathway-related genes were found to be up-regulated in Igf2dGGCT cells-where Igf2 and miR483 expression are enhanced-and down-regulated when miR483 is deleted in the same cell type (Fig. 5c,d).

In summary, the present study introduces miR483 as an important component of the ZBED6-Igf2 axis that is required to regulate $\operatorname{Ig} f 2$ expression. 
a Hierarchical cluster of

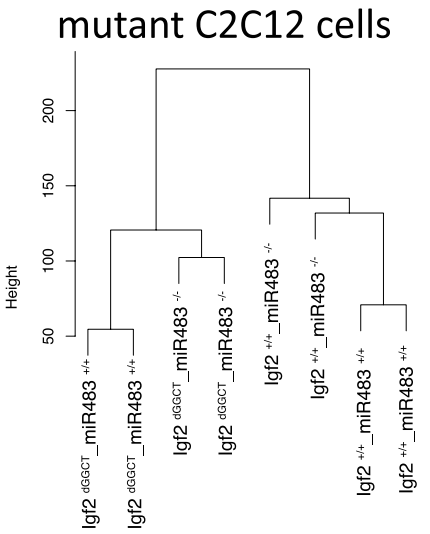

GO KEGG analysis:

C Up-reg genes in Igf2 ${ }^{d G G C T}$

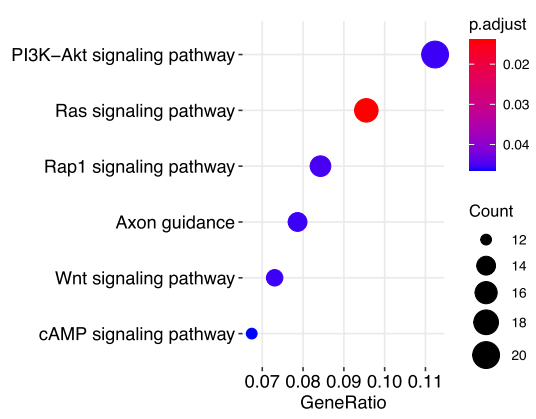

d PI3K-Akt signaling pathway

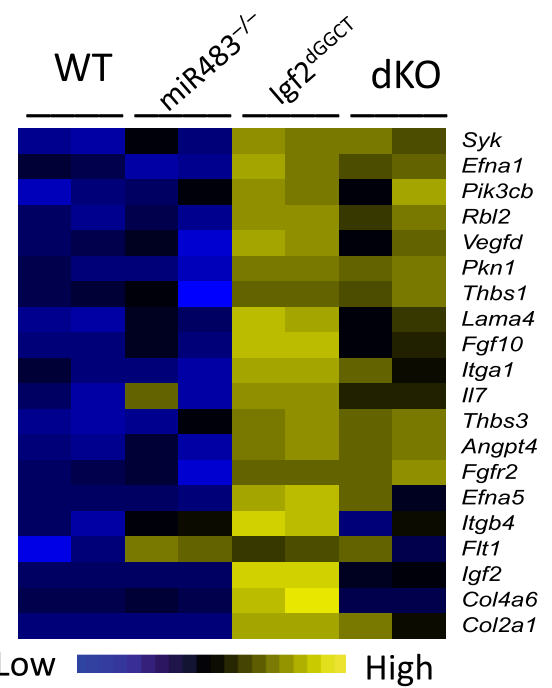

b
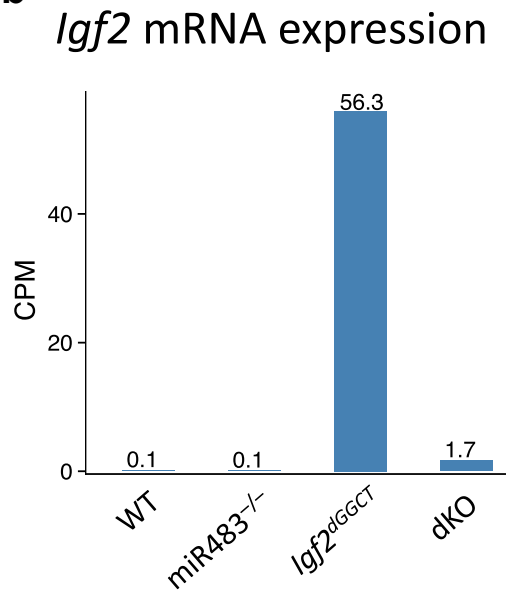

\section{Down-reg genes in dKO ${ }^{\text {mir483/dGGCT }}$ vs. Igf $2^{d G G C T}$}

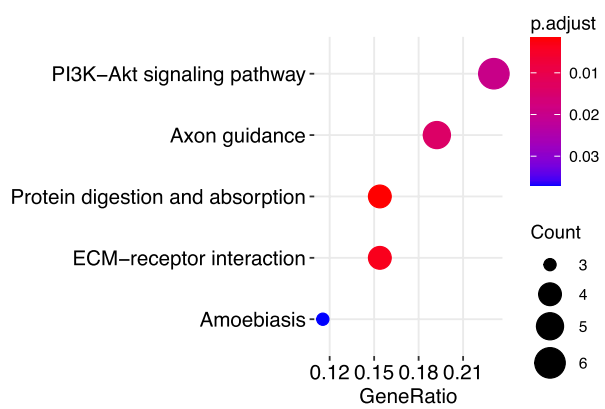

Figure 5. Comparison of RNA-seq data for wild-type, $m i R 483^{-/-}$, Igf2 ${ }^{\mathrm{dGGCT}}$ and $\mathrm{dKO} \mathrm{C} 2 \mathrm{C} 12$ cells. (a) Hierarchical cluster of mutant C2C12 based on RNA-seq data. (b) Expression of Igf2 as indicated by CPM values. (c) KEGG pathway analysis of DE genes in Igf2 ${ }^{\mathrm{dGGCT}}$ (left) and $\mathrm{dKO}^{\text {mir483/dGGCT }}$ cells (right). (d) Heatmap of genes found in the PI3K-Akt signaling pathway showing differential expression. 
$\operatorname{miR} 483^{-1-}$

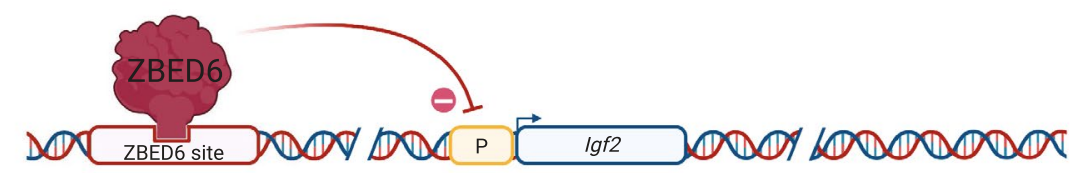

Wild-type

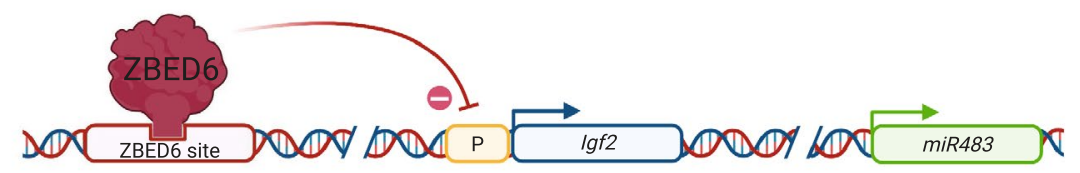

Zbed6-KO
or
Igf2 ${ }^{\mathrm{dGGCT}}$
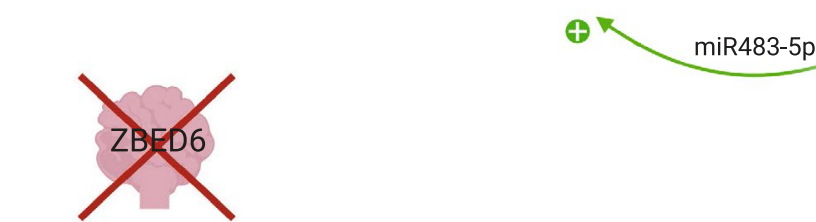

or

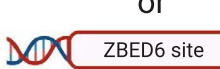

DOOP DOD
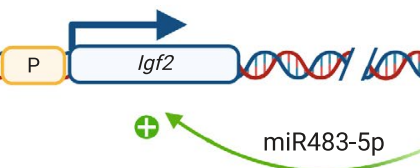

Figure 6. Schematic illustration showing the interaction between ZBED6 and the Igf2-miR483 locus and how ZBED6 suppresses expression of Igf2 and $m i R 483$, whereas miR483-5p enhances expression at the Igf2-miR483 locus. The size of hooked arrows indicates level of expression. P, promoter. Figure created with BioRender.com.

Received: 6 November 2020; Accepted: 14 September 2021

Published online: 30 September 2021

\section{References}

1. DeChiara, T. M., Robertson, E. J. \& Efstratiadis, A. Parental imprinting of the mouse insulin-like growth factor II gene. Cell 64, 849-859 (1991).

2. Sasaki, H., Ishihara, K. \& Kato, R. Mechanisms of Igf2/H19 imprinting: DNA methylation, chromatin and long-distance gene regulation. J. Biochem. 127, 711-715 (2000).

3. Van Laere, A. S. et al. A regulatory mutation in IGF2 causes a major QTL effect on muscle growth in the pig. Nature 425, 832-836 (2003).

4. Markljung, E. et al. ZBED6, a novel transcription factor derived from a domesticated DNA transposon regulates IGF2 expression and muscle growth. PLoS Biol. 7, e1000256 (2009).

5. Jiang, L. et al. ZBED6 modulates the transcription of myogenic genes in mouse myoblast cells. PLoS ONE 9, e94187 (2014).

6. Ali, M. A. et al. Transcriptional modulator ZBED6 affects cell cycle and growth of human colorectal cancer cells. Proc. Natl. Acad. Sci. 112, 7743-7748 (2015).

7. Wang, X. et al. ZBED6 negatively regulates insulin production, neuronal differentiation, and cell aggregation in MIN6 cells. FASEB J. 33, 88-100 (2019).

8. Wang, X. et al. ZBED6 counteracts high-fat diet-induced glucose intolerance by maintaining beta cell area and reducing excess mitochondrial activation. Diabetologia 64, 2292-2305 (2021).

9. Younis, S. et al. The ZBED6-IGF2 axis has a major effect on growth of skeletal muscle and internal organs in placental mammals. Proc. Natl. Acad. Sci. USA. 115, E2048-E2057 (2018).

10. Pepe, F. et al. Regulation of miR-483-3p by the O-linked N-acetylglucosamine transferase links chemosensitivity to glucose metabolism in liver cancer cells. Oncogenesis. 6, e328 (2017).

11. Cui, H. et al. IGF2-derived miR-483 mediated oncofunction by suppressing DLC-1 and associated with colorectal cancer. Oncotarget 7, 48456-48466 (2016)

12. Lupini, L. et al. Over-expression of the miR-483-3p overcomes the miR-145/TP53 pro-apoptotic loop in hepatocellular carcinoma. Oncotarget 7, 31361-31371 (2016).

13. Emmerling, V. V. et al. miR-483 is a self-regulating microRNA and can activate its own expression via USF1 in HeLa cells. Int. J. Biochem. Cell Biol. 80, 81-86 (2016).

14. Veronese, A. et al. Mutated beta-catenin evades a microRNA-dependent regulatory loop. Proc. Natl. Acad. Sci. USA. 108, 4840-4845 (2011).

15. Liu, H., French, B. A., Li, J., Tillman, B. \& French, S. W. Altered regulation of miR-34a and miR-483-3p in alcoholic hepatitis and DDC fed mice. Exp. Mol. Pathol. 99, 552-557 (2015).

16. Veronese, A. et al. Oncogenic role of miR-483-3p at the IGF2/483 locus. Cancer Res. 70, 3140-3149 (2010).

17. Liu, M. et al. The IGF2 intronic miR-483 selectively enhances transcription from IGF2 fetal promoters and enhances tumorigenesis. Genes Dev. 27, 2543-2548 (2013).

18. Patterson, E. E., Holloway, A. K., Weng, J., Fojo, T. \& Kebebew, E. MicroRNA profiling of adrenocortical tumors reveals miR-483 as a marker of malignancy. Cancer 117, 1630-1639 (2011).

19. Younis, S. et al. The importance of the ZBED6-IGF2 axis for metabolic regulation in mouse myoblast cells. FASEB J. 34(8), 10250-10266 (2020). 
20. Naito, Y., Hino, K., Bono, H. \& Ui-Tei, K. CRISPRdirect: Software for designing CRISPR/Cas guide RNA with reduced off-target sites. Bioinformatics 31, 1120-1123 (2015).

21. Dobin, A. et al. STAR: Ultrafast universal RNA-seq aligner. Bioinformatics 29, 15-21 (2013).

22. Anders, S., Pyl, P. T. \& Huber, W. HTSeq-A Python framework to work with high-throughput sequencing data. Bioinformatics 31, 166-169 (2015).

23. Robinson, M. D., McCarthy, D. J. \& Smyth, G. K. edgeR: A Bioconductor package for differential expression analysis of digital gene expression data. Bioinformatics 26, 139-140 (2010).

24. Robinson, M. D. \& Oshlack, A. A scaling normalization method for differential expression analysis of RNA-seq data. Genome Biol. 11, R25 (2010).

25. Chikina, M., Robinson, J. D. \& Clark, N. L. Hundreds of genes experienced convergent shifts in selective pressure in marine mammals. Mol. Biol. Evol. 33, 2182-2192 (2016).

26. Sergushichev, A.A., An algorithm for fast preranked gene set enrichment analysis using cumulative statistic calculation. BioRxiv. 060012 (2016)

27. Sander, J. D. \& Joung, J. K. CRISPR-Cas systems for editing, regulating and targeting genomes. Nat. Biotechnol. 32, 347-355 (2014).

28. Ran, F. A. et al. Genome engineering using the CRISPR-Cas9 system. Nat. Protoc. 8, 2281-2308 (2013).

29. Griffiths-Jones, S., Saini, H. K., van Dongen, S. \& Enright, A. J. miRBase: Tools for microRNA genomics. Nucleic Acids Res. 36, D154-D158 (2008).

30. Gu, S. \& Kay, M. A. How do miRNAs mediate translational repression? Silence 1, 11 (2010).

\section{Acknowledgements}

This project was funded by the Swedish Research Council and the Knut and Alice Wallenberg Foundation. Sequencing was performed by the SNP\&SEQ Technology Platform in Uppsala. The facility is part of the National Genomics Infrastructure (NGI) Sweden and Science for Life Laboratory. The SNP\&SEQ Platform is also supported by the Swedish Research Council and the Knut and Alice Wallenberg Foundation.

\section{Author contributions}

L.A. and S.Y. conceived the study. R.N. performed experimental and bioinformatic analysis. S.Y. contributed to the bioinformatic analysis. M.L. contributed to the experimental work. R.N., S.Y. and L.A. wrote the paper with input from M.L. All authors approved the final version before submission.

\section{Funding}

Open access funding provided by Uppsala University.

\section{Competing interests}

The authors declare no competing interests.

\section{Additional information}

Supplementary Information The online version contains supplementary material available at https://doi.org/ 10.1038/s41598-021-98777-0.

Correspondence and requests for materials should be addressed to L.A. or S.Y.

Reprints and permissions information is available at www.nature.com/reprints.

Publisher's note Springer Nature remains neutral with regard to jurisdictional claims in published maps and institutional affiliations.

Open Access This article is licensed under a Creative Commons Attribution 4.0 International License, which permits use, sharing, adaptation, distribution and reproduction in any medium or format, as long as you give appropriate credit to the original author(s) and the source, provide a link to the Creative Commons licence, and indicate if changes were made. The images or other third party material in this article are included in the article's Creative Commons licence, unless indicated otherwise in a credit line to the material. If material is not included in the article's Creative Commons licence and your intended use is not permitted by statutory regulation or exceeds the permitted use, you will need to obtain permission directly from the copyright holder. To view a copy of this licence, visit http://creativecommons.org/licenses/by/4.0/.

(c) The Author(s) 2021 